\title{
An all-thiophene electrochromic device fabricated with poly(3-methylthiophene) and poly(3,4-ethylenedioxythiophene) ${ }^{\text {th }}$
}

\author{
Shih-Wen Huang ${ }^{\mathrm{a}}$, Kuo-Chuan $\mathrm{Ho}^{\mathrm{a}, \mathrm{b}, *}$ \\ ${ }^{a}$ Department of Chemical Engineering, National Taiwan University, Taipei 10617, Taiwan \\ ${ }^{\mathrm{b}}$ Institute of Polymer Science and Engineering, National Taiwan University, Taipei 10617, Taiwan
}

Received 30 November 2004; accepted 13 February 2005

Available online 20 June 2005

\begin{abstract}
A novel all-organic electrochromic device (ECD) is presented. By electrodepositing poly (3-methylthiophene) (PMeT) in boron fluoride ethyl ether (BFEE), a strong Lewis acid, a good-quality PMeT film was obtained. On the basis of studies of PMeT, it can be regarded as a pseudo-anodic coloring material for ECDs. On the other hand, poly(3,4-ethylenedioxythiophene) (PEDOT) is an ideal cathodic coloring electrochromic material known for its high optical contrast, long-term stability, and high coloration efficiency. By combining these two thiophene derivatives, the application potential of this device was determined. The color of the ECD switches between deep blue at $-1.4 \mathrm{~V}$ (PEDOT vs. PMeT) and light red at 0.6 V. The device exhibits stable electrochromic performance, with a maximum optical attenuation $\left(\Delta T_{\max }\right)$ at $655 \mathrm{~nm}$ reaching $46 \%$ (from $9 \%$ to $55 \%$ ), and achieves a high coloration efficiency of $336 \mathrm{~cm}^{2} /$ C. After 100 repeated cycles, the cell still retained at $91.3 \%$ of its $\Delta T_{\max }$ at $655 \mathrm{~nm}$. (C) 2005 Elsevier B.V. All rights reserved.
\end{abstract}

Keywords: Coloration efficiency; Cycling stability; Electrochromic device; Poly(3,4-ethylenedioxythiophene); Poly(3-methylthiophene)

\footnotetext{
${ }^{2}$ Paper presented at the Sixth International Meeting on Electrochromism (IME-6), Brno, Czech Republic, August 29 September 2, 2004.

*Corresponding author. Department of Chemical Engineering, National Taiwan University, Taipei 10617, Taiwan. Tel.: +886223660739 ; fax: +886223623040 .

E-mail address: kcho@ntu.edu.tw (K.-C. Ho).
} 


\section{Introduction}

Electrochromism is an electrochemically induced persistent but reversible color change, and an electrochromic device is essentially an electrochemical cell in which the electrochromic electrode is separated by an electrolyte from the charge-balancing counter electrode. Thus, electrochromism can be used to regulate the throughput of radiant energy of a window.

Smart windows and large-area display devices, based on reversibly colored electrochromic materials, have become the subject of many studies around the world because of their potential applications in buildings and automobiles. Most of the electrochromic devices based on solid components have a multilayered structure sandwiched between two substrates. The successful fabrication of such a device has been achieved through overcoming quite a few technological challenges, as each of the component layers has to be optimized for its optical, electrical, and electrochromic properties before practical performance can be realized.

Electrochromic devices are of great interest as an emerging technology, especially regarding large-area smart windows capable of varying the throughput of radiant energy under the action of a voltage pulse. Smart windows are characterized by their ability to vary the radiant energy throughput. This function is achieved by incorporating chromogenic materials in the window, in most cases in the form of a thin film having electrochromic properties. Many electrochromic device designs have been proposed during the past several years as reviewed by Granqvist et al. [1-3].

During the last two decades, conducting polymers have received increasing attention because of the possibility of controlling their electrical conductivity between an insulating and a conducting state. Conducting polymers, such as polythiophene and its derivatives, are promising industrial materials because of recent progress in their processing. Applications of poly(3-alkylthiophenes) are found in lithium secondary batteries and also in ECDs [4]. Poly(3-methylthiophene) (PMeT) has extensively been researched and even used for a long time in photovoltaic cells, solid-state lithium cells, electrochemical sensors, and modified electrodes [5]. In addition, much research has revealed that PMeT is a promising electrochromic material [6,7]. Applications of PMeT electrodeposited in nitrobenzene for ECDs have been proposed [8-10], and an all-thiophene ECD was reported by Ribeiro et al. [11]. PMeT belongs to a class of conjugated polymers with non-degenerated ground state symmetry and is a high-conducting polymer. It has been demonstrated, for instance, that PMeT has interesting electrochromic properties with a response time of a few milliseconds and a cyclability of more than $10^{5}$ cycles [12].

Recent interest in these simple alkyl thiophenes has dwindled due to their high oxidation potential, which results in the irreversible over-oxidation of the polymer electrodeposited onto the electrode. Polythiophenes deposited on indium tin oxide (ITO) substrate from common organic solvents are of low quality and are poor conductors because of their high oxidation potentials. Although thiophene oligomers can be electrochemically polymerized at lower potentials than the parent monomer, it was found that the resulting polymers exhibit low-average conjugation lengths and 
poor mechanical properties. Shi et al. [13] developed a new electrochemical polymerization method for high-oxidation potential monomers in boron fluoride ethyl ether (BFEE) which can yield high-conducting polythiophene films with high tensile strengths. Subsequently, the electrochromic properties and stabilities of polythiophene (PT), poly(3-methylthiophene) (PMeT), poly(3-bromothiophene), and poly(3,4-dibromothiophene), electrodeposited in BFEE, were reported by Alkan et al. [14]. They also concluded that PMeT films electrodeposited in BFEE had better electroactivity and cycling stability properties than those of the other three. Two other thiophene derivatives electrodeposited in BFEE, bithiophene and terthiophene, were reported by Zhang et al. [15,16].

Poly(3,4-ethylenedioxythiophene) (PEDOT) exhibits high color contrast, changing from transmissive sky-blue in the oxidized state to deep-blue in the reduced state. The favorable electrochromic properties of PEDOT are currently the focus of a large portion of research in the field [17]. PEDOT has been utilized in several ways, including sensors [18,19], capacitors [20], and hole-transporting layer applications [21].

The objective of this paper was to develop a novel all-thiophene ECD. The aim was to investigate and report on the feasibility of using both PMeT and PEDOT as components in an ECD with vivid color contrast.

\section{Experimental}

\subsection{Chemicals and substrate}

Reagent grade acetonitrile (ACN), propylene carbonate (PC), and boron fluoride ethyl ether (BFEE) were purchased from Lancaster, Tedia and Fluka, respectively. ACN was dehydrated before being used by $4-\AA$ molecular sieves from Acros. Tetran-butyl ammonium perchlorate $\left(\mathrm{TBAClO}_{4}\right), 99 \%$, and tetra-butyl ammonium fluoroborate $\left(\mathrm{TBABF}_{4}\right), 99 \%$, were both purchased from Fluka. The monomers, 3-methylthiophene (MeT), 98\%, and 3,4-ethylenedioxythiophene (EDOT), were purchased from Aldrich. $\mathrm{TBAClO}_{4}, \mathrm{TBABF}_{4}$, and the monomers were used without further purification.

\subsection{Electrochromic polymers preparation}

Polymer films were obtained by constant potential polymerization at potentials slightly higher than the monomer oxidation onset potential in order to obtain a homogeneous thin film on the ITO electrodes. The electrochemical polymerization of PMeT and PEDOT by potentiostatic methods was carried out on ITO as a working electrode, a platinum sheet as a counter electrode, and $\mathrm{Ag} / \mathrm{Ag}^{+}$as a reference electrode. All potentials reported in the following are relative to $\mathrm{Ag} / \mathrm{Ag}^{+}$, which contained a solution of $0.01 \mathrm{M} \mathrm{AgNO}_{3}$ and $0.1 \mathrm{M} \mathrm{TBAClO}_{4}$ in acetonitrile.

The ITO substrates (sheet resistance $=20 \Omega /$ sq.) were supplied by RiTdisplay Corporation (Hsinchu Industrial Park, Taiwan). The ITO substrates were treated prior to use by sonication in $\mathrm{HCl}_{(\mathrm{aq})}$ for $3 \mathrm{~min}$ and $\mathrm{DI}$ water for another $3 \mathrm{~min}$. The 
ITO surfaces were kept clean, and an epoxy spacer $(3 \mathrm{M})$ was used to control the electroactive area $(2 \times 2 \mathrm{~cm})$. For better uniformity of current supply, copper tape was applied on one edge of each ITO substrate.

PMeT was electrodeposited in BFEE containing $0.01 \mathrm{M}$ MeT monomer at a constant potential of $1.5 \mathrm{~V}$ with a charge density of $20 \mathrm{mC} / \mathrm{cm}^{2}$ (and an average thickness of $210 \mathrm{~nm}$ ). The PMeT thin film electrodes were removed from the monomer/electrolyte solution after electrochemical polymerization and rinsed with ethanol to produce clean surfaces without monomer.

PEDOT was electrodeposited in ACN which contained 0.01 M EDOT monomer and $0.1 \mathrm{M} \mathrm{TBABF} 4$ at a constant potential of $1.2 \mathrm{~V}$ with a charge density of $20 \mathrm{mC} / \mathrm{cm}^{2}$ (and an average thickness of $120 \mathrm{~nm}$ ). After forming PEDOT thin films, the electrodes were rinsed with pure $\mathrm{ACN}$ to remove the monomer on the film surfaces.

\subsection{Liquid electrolyte composition}

The electrolytic solution was composed of $0.1 \mathrm{M} \mathrm{TBABF}_{4}$ in $\mathrm{PC}$ and the solution was dehydrated by $4-\AA$ molecular sieves.

\subsection{Device assembly}

Before assembling, PMeT and PEDOT were cycled from -0.4 to $0.8 \mathrm{~V}$ and -1.2 to $0.3 \mathrm{~V}$ for three cycles (at a scan rate of $100 \mathrm{mV} / \mathrm{s}$ ) and pretreated to their undoped and doped states, respectively. The ECD was assembled by sandwiching a doped PEDOT electrode and an undoped PMeT electrode, separated by a $280-\mu \mathrm{m}$ spacer and a $0.1 \mathrm{M} \mathrm{TBABF}_{4}$-based liquid electrolyte.

\subsection{Instrumentation}

An EG\&G model 273A potentiostat/galvanostat was used for PMeT electrodeposition on ITO. Except for this, all other electrochemical experiments were carried out using an Eco-Chemie Autolab PGSTAT30. UV-vis-NIR results, including in situ spectroelectrochemical measurements, were obtained using a Shimadzu model UV-1601PC spectrophotometer. Thicknesses of polymer thin films were measured with a profilometer (Sloan Dektak, model 3030).

\subsection{Electrochemical and spectral measurements}

The PMeT and PEDOT thin-film electrodes were characterized electrochemically in $0.1 \mathrm{M} \mathrm{TBABF} 4$ plus PC by the three-electrode cyclic voltammetry $(\mathrm{CV})$ method and in situ spectral measurements. The transmittance spectra of the ECD were measured at different equilibrated applied voltages (PEDOT vs. PMeT). The applied voltage was $0.6 \mathrm{~V}$ for $20 \mathrm{~s}$ and was changed to $-1.4 \mathrm{~V}$ for $20 \mathrm{~s}$ in each cycle. The transmittance spectra at $655 \mathrm{~nm}$ were continuously recorded in situ for 100 cycles. 


\section{Results and discussion}

The electrochemical p-doping process, which makes the conjugated polymers conductive, is an oxidation process, and the undoping process corresponds to the reduction reaction which returns the material to the insulating state. The overall process, which involves ion diffusion into and out of the polymer matrix to balance the charge, can be represented as Eqs. (1) and (2) for PMeT and PEDOT, respectively,

$$
\begin{array}{ccc}
(\mathrm{MeT})_{n}+n y\left(\mathrm{BF}_{4}^{-}\right) & \leftrightarrow & {\left[\left(\mathrm{MeT}^{+}\right)+y\left(\mathrm{BF}_{4}^{-}\right)\right]_{n}+n y \mathrm{e}^{-}} \\
\text {light red } & \text { deep blue }
\end{array}
$$

and

$$
\begin{array}{ccc}
{\left[\left(\mathrm{EDOT}^{+}\right)+y\left(\mathrm{BF}_{4}^{-}\right)\right]_{n}+n y \mathrm{e}^{-}} & \leftrightarrow & (\mathrm{EDOT})_{n}+n y \mathrm{BF}_{4}^{-} \\
\text {light blue } & \text { purple }
\end{array}
$$

where $n$ is the number of repeated units and $y$ is the stoichiometric number of the counter ion.

\subsection{Electrochemical and spectral properties of PMeT and PEDOT thin-film electrodes}

Because of the inductive effect of the methyl substitute, MeT polymerized in BFEE at a lower potential than thiophene. Although the role of BFEE in the doping process during electropolymerization is not fully established, it has been proposed that it facilitates electrochemical polymerization by lowering the aromatic resonance energy and promoting abstraction of an electron from the $\alpha$-position of the heterocyclic ring. The stability of PMeT electrodeposited in BFEE is reasonably good. Testing was carried out in a PC solution containing $0.1 \mathrm{M} \mathrm{TBABF}$. Fig. 1 shows the charge capacity variations of both PMeT and PEDOT thin-film electrodes as a function of cycle number while performing CV from the 1st to the 1000th cycle (at a scan rate of $100 \mathrm{mV} / \mathrm{s}$ ). The potential range of $\mathrm{CV}$ was between -0.4 and $0.8 \mathrm{~V}$ in consideration of the best visual contrast for PMeT. The same test procedure for the PEDOT thin-film electrode ranged between 0.3 and $-1.2 \mathrm{~V}$.

In Fig. 1, the charge capacity of PMeT increased during the first 20 cycles because a PMeT thin film which was electrodeposited in BFEE without anion doping takes time to reach equilibrium during the initial ion doping and dedoping processes. For PEDOT, however, there is no increase in the charge capacity for the first few cycles because the doped ion within the PEDOT thin film was the same as the one in the solution. Both PMeT and PEDOT showed a continuous decay in the last few hundred cycles for the reasons which might have to do with impurities in the electrolyte solution, the unsuitable potential window, incompletion of the doping and dedoping processes, and irregularity of the polymer chains used.

Like most other poly alkylthiophenes, PMeT in situ spectra at different potentials overlapped between 550 and $650 \mathrm{~nm}$ [4]. These show the intrinsic spectral properties 


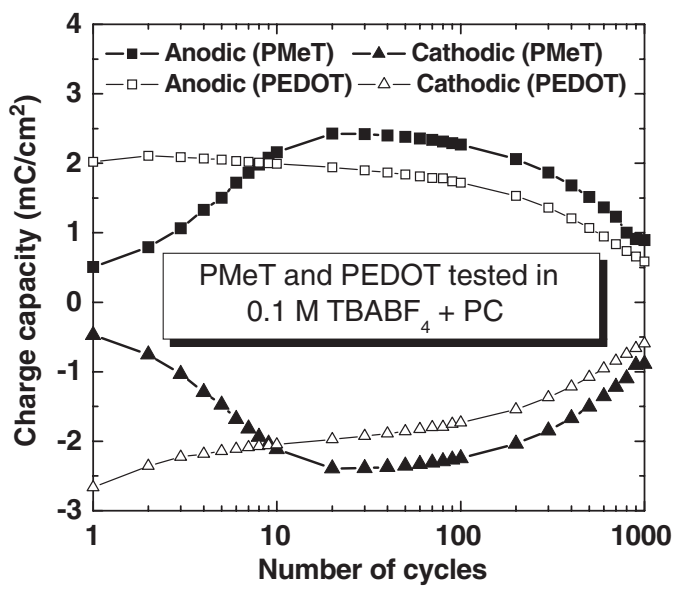

Fig. 1. The variations of the charge capacities of both PMeT and PEDOT thin films as a function of cycle number. The $\mathrm{CV}$ was scanned from -0.4 to $0.8 \mathrm{~V}$ for PMeT and from 0.3 to $-1.2 \mathrm{~V}$ for PEDOT at a scan rate of $100 \mathrm{mV} / \mathrm{s}$ in $\mathrm{PC}$ containing $0.1 \mathrm{M} \mathrm{TBABF}_{4}$.

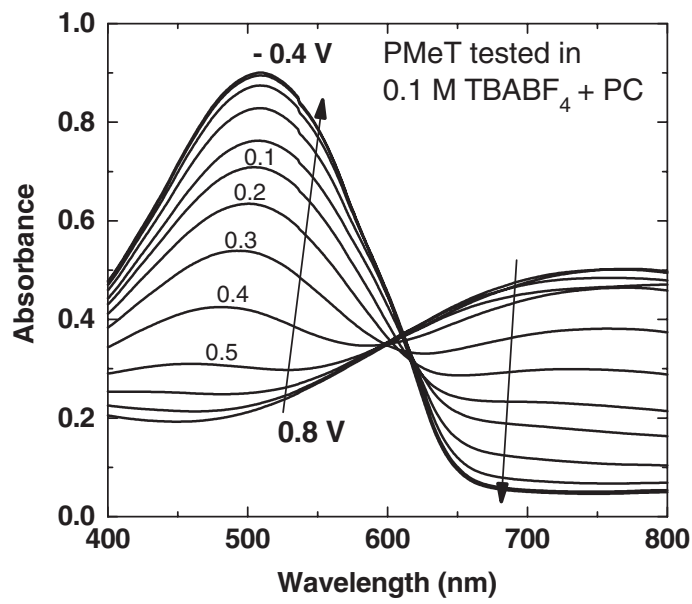

Fig. 2. In situ UV-vis absorption spectra of PMeT switched in $0.1 \mathrm{M} \mathrm{TBABF} 4 \mathrm{PC}$ solution at different equilibrium potentials: $E=-0.4$ to $0.8 \mathrm{~V}$ (vs. $\mathrm{Ag} / \mathrm{Ag}^{+}$). The spectral data show a potential difference of $0.1 \mathrm{~V}$ for the two nearest neighboring curves.

of poly alkylthiophenes which have no colorless state. The equilibrium spectra obtained at different potentials from -0.4 to $0.8 \mathrm{~V}$ (vs. $\mathrm{Ag} / \mathrm{Ag}^{+}$) are shown in Fig. 2. At $-0.4 \mathrm{~V}$, the characteristic peak of the spectrum was at $510 \mathrm{~nm}$. As the voltage increased, an isosbestic point was observed at $610 \mathrm{~nm}$. Also, the equilibrium spectra of PEDOT at different potentials from 0.3 to $-1.2 \mathrm{~V}$ (vs. $\mathrm{Ag} / \mathrm{Ag}^{+}$) are shown in Fig. 3. The characteristic peak of the spectrum at $-1.2 \mathrm{~V}$ was observed at around 


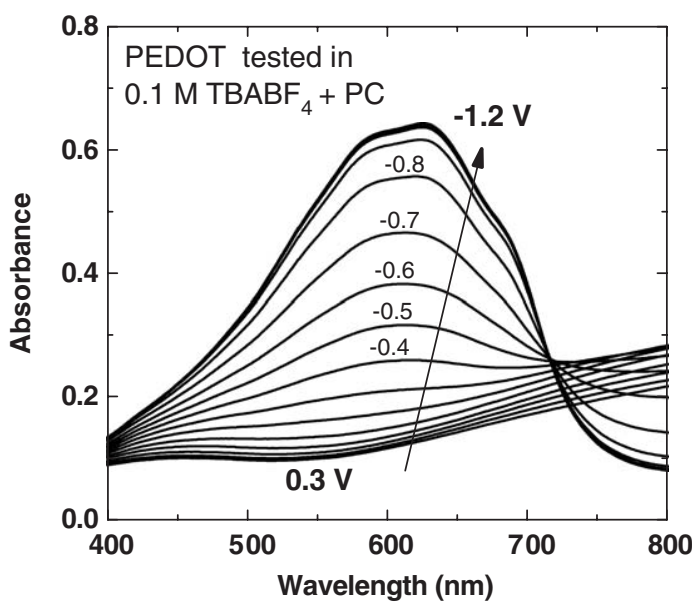

Fig. 3. In situ UV-vis absorption spectra of PEDOT switched in $0.1 \mathrm{M} \mathrm{TBABF}_{4} \mathrm{PC}$ solution at different equilibrium potentials: $E=0.3$ to $-1.2 \mathrm{~V}$ (vs. $\mathrm{Ag} / \mathrm{Ag}^{+}$). The spectral data show a potential difference of $0.1 \mathrm{~V}$ for the two nearest neighboring curves.

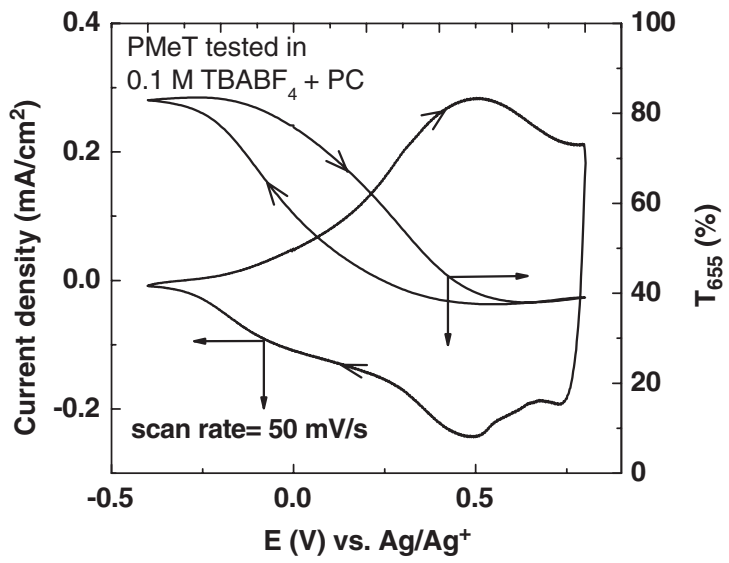

Fig. 4. In situ current-transmittance measurements at $655 \mathrm{~nm}$ for a PMeT thin film in a CV tested in $0.1 \mathrm{M}$ $\mathrm{TBABF}_{4}+\mathrm{PC}$ at a scan rate of $50 \mathrm{mV} / \mathrm{s}$.

$600 \mathrm{~nm}$, and the isosbestic point was also noted at $715 \mathrm{~nm}$ as the voltage was increased to $-0.4 \mathrm{~V}$.

PMeT showed a reversible color change during cyclic potential sweeping between -0.4 and $0.8 \mathrm{~V}$. The in situ transmittance of PMeT at $655 \mathrm{~nm}$ while performing CV cycling (at a scan rate of $50 \mathrm{mV} / \mathrm{s}$ ) is shown in Fig. 4. The doping process was faster than the undoping one, as observed by the transmittance lag.

The typical CV results of PEDOT and PMeT in $0.1 \mathrm{M} \mathrm{TBABF}_{4}$ are shown in Fig. 5. The oxidized state of PEDOT is light blue $(0.3 \mathrm{~V})$ and the reduced state is deep purple $(-1.2 \mathrm{~V})$; the oxidized state of PMeT is blue $(0.8 \mathrm{~V})$ and the reduced state 


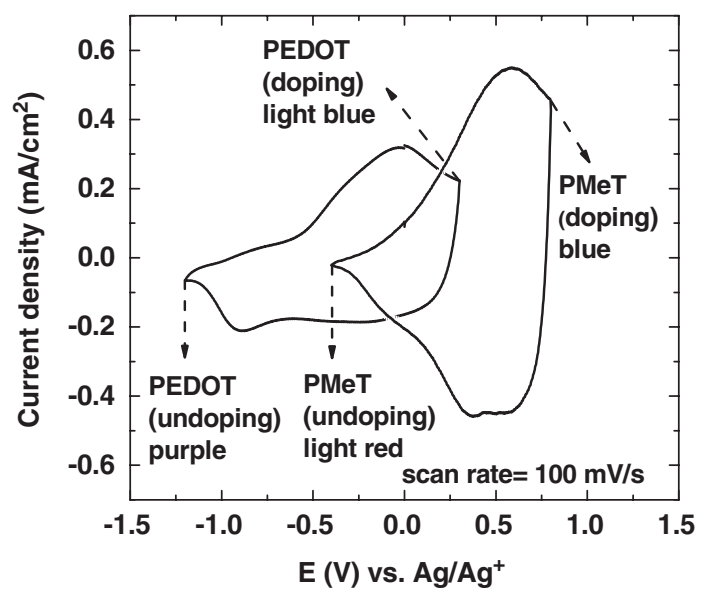

Fig. 5. Typical CVs of PEDOT and PMeT in $0.1 \mathrm{M} \mathrm{TBABF}_{4}+\mathrm{PC}$ at a scan rate of $100 \mathrm{mV} / \mathrm{s}$.

is red $(-0.4 \mathrm{~V})$. When the polymers, PEDOT and PMeT, change from neutral to a doped state, charge carriers are generated first as polarons, then bipolarons, and finally a phase transformation into the metallic state occurs. Each of these steps involves a one-electron transfer [22].

Both PMeT and PEDOT typically show two reduction peaks and at least one oxidation peak in their CV results. Chen and Inganäs [22] pointed out that there might be a three-step redox process, and they obtained three reduction peaks and two oxidation peaks at a very fast scan rate of $40 \mathrm{~V} / \mathrm{s}$ for PEDOT. They also extended the redox transformation to PMeT. In fact, only one broad oxidation peak, not the two expected, was observed. This was explained by Chen and Inganäs as the unclear individual oxidation processes being due to the presence of unstable intermediate or the very close potential overlap. The observation of a broad reduction peak is reasonable because the three reduction peaks have almost the same height.

The transmittance spectra of the colored and bleached states of PMeT and PEDOT are shown in Fig. 6(a) and (b), respectively. It is expected that the optical complementary wavelength of the ECD will fall in the red region.

\subsection{Coloration efficiencies of PMeT and PEDOT thin-film electrodes}

The relationships between the optical density change $(\Delta \mathrm{OD})$ at $655 \mathrm{~nm}$ and charge density, for both PMeT and PEDOT electrodes, are shown in Figs. 7 and 8, respectively. For PMeT thin-film electrodes, the relationship was determined by switching voltages between a fixed bleached state $(-0.4 \mathrm{~V})$ and different colored states $(-0.1,0,0.1,0.2,0.3,0.4,0.5,0.6,0.7$, and $0.8 \mathrm{~V})$; for PEDOT, the switching voltages were between a fixed bleached state $(0.3 \mathrm{~V})$ and different colored states $(0.2$ to $-1.2 \mathrm{~V}$, with a decrement of $0.1 \mathrm{~V}$ ). Each voltage step lasted for $60 \mathrm{~s}$ to allow the reaction to reach equilibrium. Integration of the $I-t$ curve (with a recorded time 


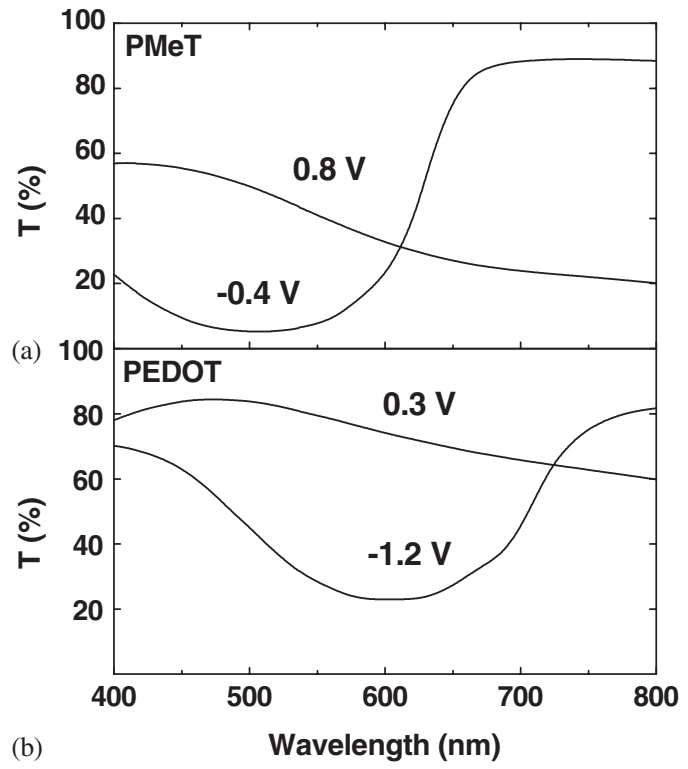

Fig. 6. Transmittance spectra at the bleached and the darkened states for (a) PMeT $(-0.4 \mathrm{~V}$ bleached; $0.8 \mathrm{~V}$ colored), and (b) PEDOT ( $0.3 \mathrm{~V}$ bleached; $-1.2 \mathrm{~V}$ colored).

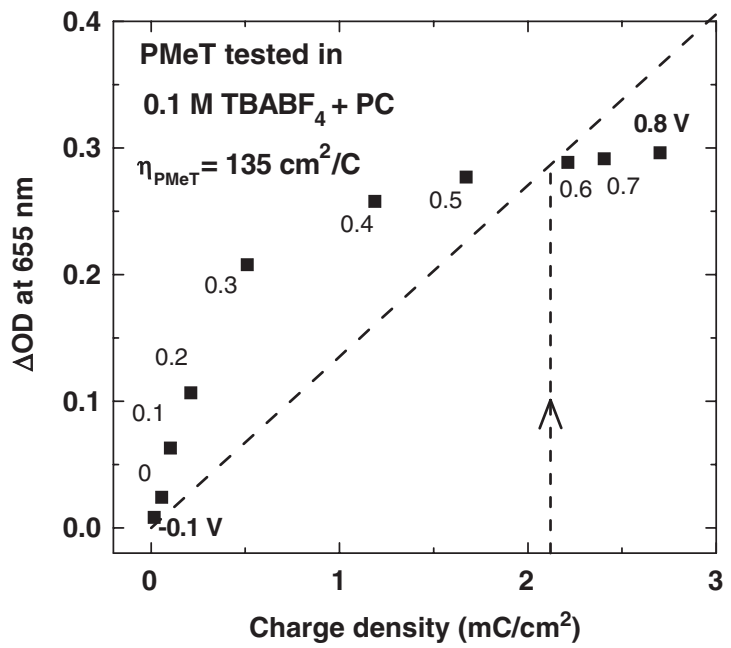

Fig. 7. The optical density change of a PMeT thin film at $655 \mathrm{~nm}$ as a function of the inserted charge density.

interval of $0.025 \mathrm{~s}$ ) shows the inserted charge density for each step, and the relationship between $\triangle \mathrm{OD}$ and the charge density was obtained. However, the real coloration efficiencies of PMeT and PEDOT depended on the operating voltage. According to the effective charge density of the ECD (at about $2.12 \mathrm{mC} / \mathrm{cm}^{2}$ ) which 


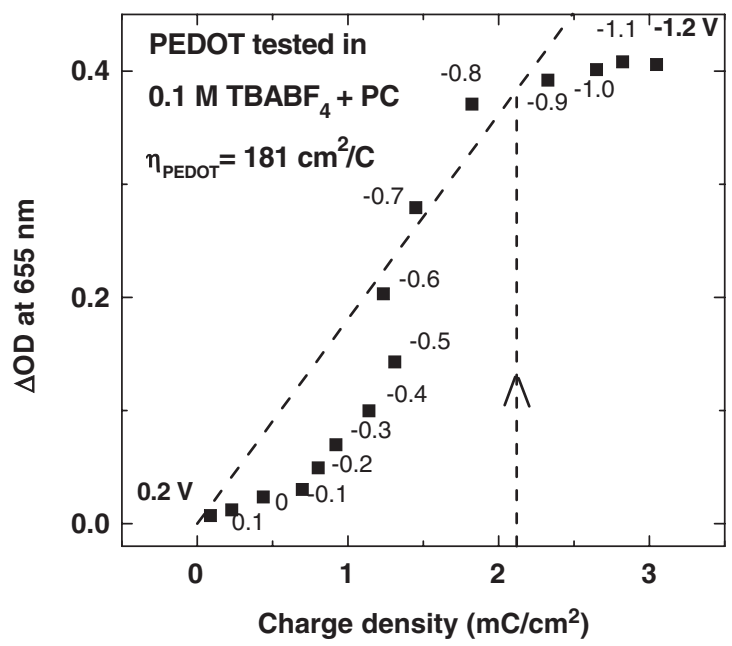

Fig. 8. The optical density change of a PEDOT thin film at $655 \mathrm{~nm}$ as a function of the inserted charge density.

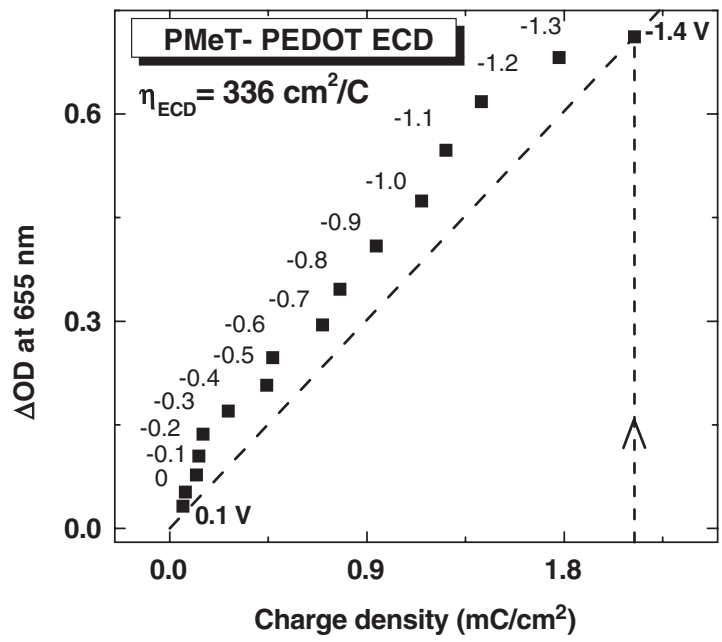

Fig. 9. The optical density change of the PMeT-PEDOT ECD at $655 \mathrm{~nm}$ as a function of the inserted charge density.

is verified in Fig. 9, the coloration efficiencies for both PMeT and PEDOT thin-film electrodes were calculated based on the same passed charge density of $2.12 \mathrm{mC} / \mathrm{cm}^{2}$ at each electrode. For PMeT, the coloration efficiency was $135 \mathrm{~cm}^{2} / \mathrm{C}$ with a passed charge density of $2.12 \mathrm{mC} / \mathrm{cm}^{2}$, which corresponds to the potential steps from -0.4 to $0.6 \mathrm{~V}$; for PEDOT, the coloration efficiency was $181 \mathrm{~cm}^{2} / \mathrm{C}$ with a passed charge density of $2.12 \mathrm{mC} / \mathrm{cm}^{2}$, which corresponds to the potential steps from 0.3 to $-0.9 \mathrm{~V}$. 
The coloration efficiencies of many conjugated polymers are not constant. The reason for the nonlinear graphs (Figs. 7-9) is that conjugated polymers possess different states while being doped. Polarons, bipolarons, and $\pi$-dimers all factor into the overall properties of organic electrochromic polymers. Therefore, the task of measuring the coloration efficiencies of such systems becomes complex. To simplify this electrochromic property, we have reported the coloration efficiencies of PMeT and PEDOT based on the consumed charge density in the PMeT-PEDOT ECD. Similar approach was developed by Gaupp et al. [23], who reported a method to quantify the composite coloration efficiency of organic electrochromic polymers at $95 \%$ of the total $\Delta$ OD measured at $\lambda_{\max }$.

\subsection{Coloration efficiency of the ECD}

The coloration efficiency of the ECD measured at $655 \mathrm{~nm}$ is shown in Fig. 9. The applied potential was fixed at $0.6 \mathrm{~V}$ upon bleaching and then switched to different potentials $(0.1$ to $-1.4 \mathrm{~V}$, with a decrement of $0.1 \mathrm{~V})$ upon coloring for an interval of $60 \mathrm{~s}$. Integration of the $I-t$ curve (with a recorded time interval of $0.025 \mathrm{~s}$ ) shows the inserted charge density for each step. The relationship between $\Delta \mathrm{OD}$ and the charge density was thus obtained. The calculated coloration efficiency of the ECD was $336 \mathrm{~cm}^{2} / \mathrm{C}$, as indicated by the slope of the dashed line in Fig. 9. This value is close to the sum of the coloration efficiencies of PMeT $\left(135 \mathrm{~cm}^{2} / \mathrm{C}\right)$ and that of the PEDOT $\left(181 \mathrm{~cm}^{2} / \mathrm{C}\right)$. In addition, the coloring and bleaching potentials applied to the ECD were also confirmed by the corresponding input charge densities of both thin-film electrodes and the ECD. Theoretically speaking, the bleaching and coloring potentials of the ECD could be expressed by the bleaching and coloring potentials of both electrodes: $E_{\text {bleach,ECD }}=E_{\text {bleach,PEDOT }}-E_{\text {bleach,PMeT }}=(0.3 \mathrm{~V})-(-0.4 \mathrm{~V})=0.7 \mathrm{~V}$, and $E_{\text {color,ECD }}=E_{\text {color,PEDOT }}-E_{\text {color,PMeT }}=(-0.9 \mathrm{~V})-(0.6 \mathrm{~V})=-1.5 \mathrm{~V}$. The calculated coloring and bleaching potentials were very close to the applied potentials of the $\mathrm{ECD}\left(E_{\text {bleach,ECD }}=0.6 \mathrm{~V}\right.$ and $\left.E_{\text {color,ECD }}=-1.4 \mathrm{~V}\right)$.

\subsection{Spectral characterization and $C V$ of the ECD}

The transmittance spectra of the ECD are shown in Fig. 10. By applying a potential of $0.6 \mathrm{~V}$ (PEDOT vs. PMeT), the ECD reached its bleached state; however, the ECD reached its colored state when $-1.4 \mathrm{~V}$ (PEDOT vs. PMeT) was applied. According to Fig. 10, the maximum optical attenuation difference occurred at $655 \mathrm{~nm}$, or the red region. The in situ transmittances of the ECD were measured at $655 \mathrm{~nm}$ with step potentials of 0.6 and $-1.4 \mathrm{~V}$. Fig. 11 shows the CVs of the device in the potential range. It shows that no other side reaction occurred during cycling, and that the operating potential window is safe for the ECD.

\subsection{Cycling stability of the ECD}

Fig. 12 shows the device's transmittance in response to the potential being repeatedly switched between 0.6 and $-1.4 \mathrm{~V}$ (20 s for each). The solid line corresponds 


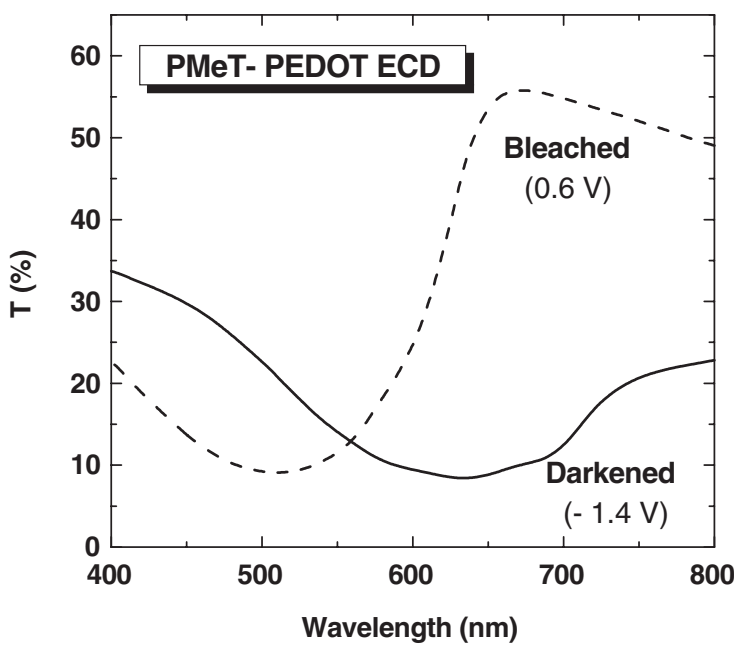

Fig. 10. Transmittance spectra of the ECD switched at $+0.6 \mathrm{~V}$ for bleaching and $-1.4 \mathrm{~V}$ for darkening (dash line: bleached state; solid line: colored state).

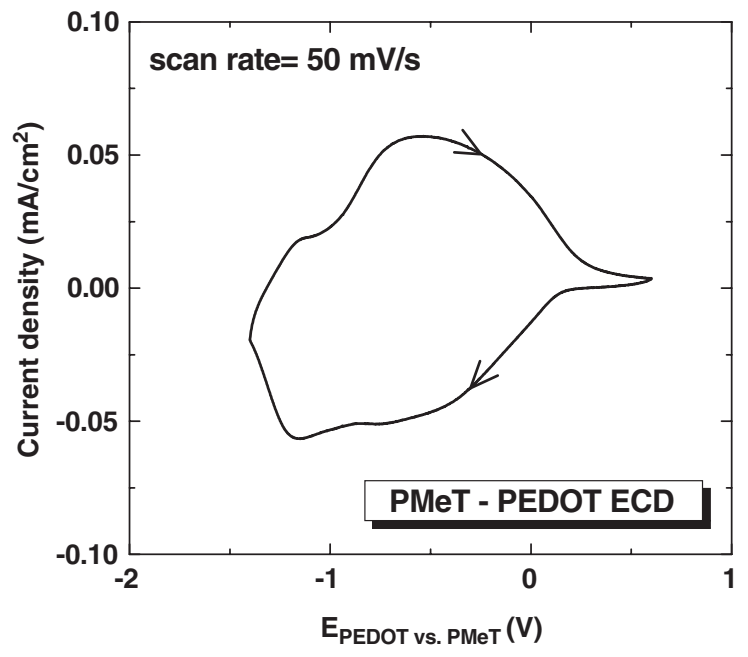

Fig. 11. The CV for the PMeT-PEDOT ECD at a scan rate of $50 \mathrm{mV} / \mathrm{s}$.

to the first five cycles, and the dashed line corresponds to the 95th-100th cycles. It was found that the response of the ECD was very fast (less than $1 \mathrm{~s}$ ) and that the ECD was stable. After 100 cycles, $\Delta T$ decreased from $46 \%$ to $42 \%$. The ECD still remained at $91.3 \%$ of its initial $\Delta T$ value. As for the switching instability, there might be some reasons for the decay: the impurities (oxygen, water, etc,) in the electrolyte solution, the irregularities of polymer thin films, the incompletion of doping and dedoping processes, and the possible over-potentials during potential steps operation. 


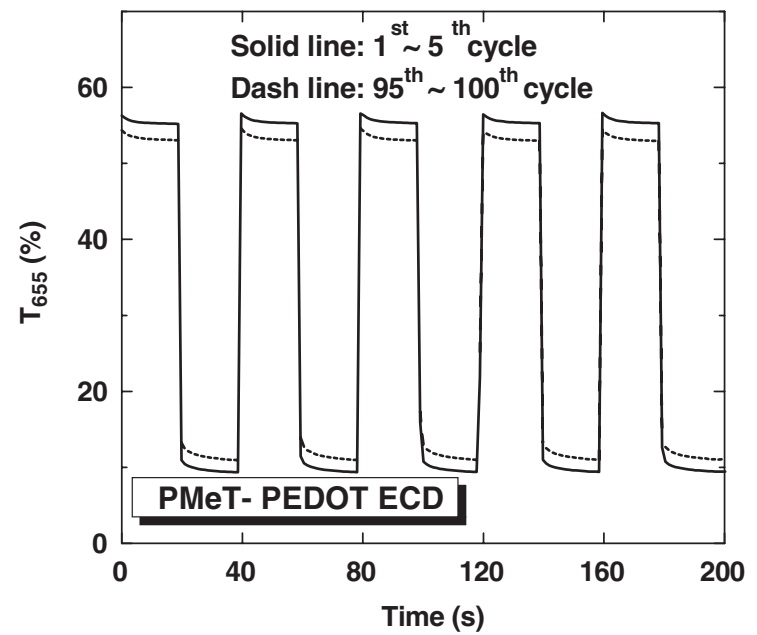

Fig. 12. In-situ transmittance response of the PMeT-PEDOT ECD during repeated potential steps switched between 0.6 and $-1.4 \mathrm{~V}$ (solid line: 1st-5th cycle; dash line: 95 th-100th cycle).

\subsection{Comparison of PMeT-based or all-thiophene ECDs in the literature}

A literature review revealed that PMeT-based ECDs have not been widely studied because of their limited optical performance and electro-optical stability. Similarly, all-thiophene ECDs seldom exhibit good optical contrast or high transmittance attenuation because of their intrinsic optical characteristics. However, our first attempt showed that the PMeT-PEDOT coupling is a novel, all-organic ECD. By electrodepositing PMeT on ITO in BFEE, the improvement in the electrochromic properties would make this combination worthy of further exploration. A comparison between the performance of this ECD and other reported PMeT-based and all-thiophene ECDs is given in Table 1. By comparing the performances of the PMeT-PEDOT ECD to previous ECDs made with PMeT as a primary electrochromic electrode reported in the literature, it was found that the novel configuration of the PMeT-PEDOT couple offered much higher optical attenuation at $655 \mathrm{~nm}$ with significantly better cycling stability. Although the performance of this ECD is acceptable, as compared with other known PMeT-based and all-thiophene ECDs, a lot of work to further study for its long-term stability, at-rest characteristics, and environmental stability is required.

\section{Conclusions}

Conjugated polymers are very promising electrochromic materials. The great variety of colors offered by conjugated polymers is the most significant characteristic of this class of ECDs in comparison with other inorganic ECDs. 
Table 1

A partial list of PMeT-based and all-thiophene ECDs reported in literature

\begin{tabular}{|c|c|c|c|c|}
\hline $\begin{array}{l}\text { Cell configuration substrate/ } \\
\mathrm{EC} 1 / \text { electrolyte/EC2/substrate }\end{array}$ & $\begin{array}{l}\text { Operation } \\
\text { voltage }\end{array}$ & $\begin{array}{l}\text { Bleach } / \text { color } \\
\text { states } \Delta T_{\mathrm{b}} / T_{\mathrm{d}} \\
\text { and } \Delta T(\%)\end{array}$ & Stability & Ref. \\
\hline ITO/Electrolyte ${ }^{1} / \mathrm{PMeT}^{\mathrm{a}} /$ ITO & $-0.3-2.5 \mathrm{~V}$ & $\begin{array}{l}41 / 635 \% \text { at } \\
530 \mathrm{~nm}\end{array}$ & $\begin{array}{l}\text { After } 1000 \\
\text { cycles, no color } \\
\text { changed }\end{array}$ & {$[8]$} \\
\hline $\begin{array}{l}\mathrm{ITO}^{\mathrm{L}} \mathrm{Li}_{x} \mathrm{NiO}_{z} / \text { Electrolyte }^{1} / \\
\mathrm{PMeT}^{\mathrm{a}} / \text { ITO }\end{array}$ & $0-2.5 \mathrm{~V}$ & $\begin{array}{l}43 / 1132 \% \text { at } \\
530 \mathrm{~nm}\end{array}$ & - & [9] \\
\hline $\begin{array}{l}\text { ITO/PMeT } / \text { Electrolyte }^{2} / \\
\text { Poly(3,3-dimethyl-2,2- } \\
\text { bithiophene)/ITO }\end{array}$ & $-0.9-0.9 \mathrm{~V}$ & $\begin{array}{l}28 / 1414 \% \text { at } \\
560 \mathrm{~nm}\end{array}$ & $\begin{array}{l}\text { After } 2000 \text { cycles } \\
\Delta T \text { remains } 10 \%\end{array}$ & {$[10]$} \\
\hline $\begin{array}{l}\text { PET/ITO/PEDOT-PSS/ } \\
\text { Electrolyte }^{3} / \text { Poly }(12- \\
\text { MPDDT }^{\mathrm{c}} / \text { ITO/Glass }\end{array}$ & $0-1.7 \mathrm{~V}$ & $\begin{array}{l}68 / 2642 \% \text { at } \\
650 \mathrm{~nm}\end{array}$ & $\begin{array}{l}\text { After } 200 \text { cycles } \\
\Delta T \text { remains } 31 \%\end{array}$ & [11] \\
\hline $\begin{array}{l}\text { ITO } / \text { PMeT }^{\mathrm{b}} / \text { Electrolyte }^{4} / \\
\text { PEDOT/ITO }\end{array}$ & $-1.4-0.6 \mathrm{~V}$ & $\begin{array}{l}55 / 946 \% \text { at } \\
655 \mathrm{~nm}\end{array}$ & $\begin{array}{l}\text { After } 100 \text { cycles } \\
\Delta T \text { remains } 42 \%\end{array}$ & This work \\
\hline
\end{tabular}

Electrolyte ${ }^{1}$ : PC-1 M $\mathrm{LiClO}_{4}$.

Electrolyte 2 : PAN-PC-ethylene carbonate- $\mathrm{LiCO}_{4}$.

Electrolyte $^{3}$ : P(EO-EPI)- $\mathrm{LiClO}_{4}$.

Electrolyte ${ }^{4}:$ PC-0.1 M TBABF

PMeT $^{\mathrm{a}}$ : $0.2 \mathrm{M}$ tetrabutylammonium hexafluorophophate in nitrobenzene; $0.2 \mathrm{M}$ monomer;constant current density $=5 \mathrm{~mA} / \mathrm{cm}^{2}$; temperature $=5^{\circ} \mathrm{C}$.

$\mathrm{PMeT}^{\mathrm{b}}$ : $0.01 \mathrm{M}$ monomer in BFEE; $E=1.5 \mathrm{~V}$ (vs. $\mathrm{Ag} / \mathrm{Ag}^{+}$); room temp.

Poly(12-MPDDT) $)^{\mathrm{c}}$ : poly\{3-[12-(p-methoxyphenoxy)dodecyl]thiophene\}.

An all-thiophene ECD made of PEDOT and PMeT thin films with a liquid electrolyte composed of $\mathrm{TBABF}_{4}$ in propylene carbonate (PC) was successfully fabricated. The device's transmittances, measured as $T_{655}(\%)$, ranged from $9 \%$ to $55 \%$. The device can be switched electrochemically between deep blue and transmissive light red. After 100 cycles, the maximum optical attenuation at $655 \mathrm{~nm}$ still remained at $91.3 \%$ of its initial value, making this ECD a promising candidate for further study.

So far, the ECD performed fairly well even though it has not been optimized. Sealing problems can be improved by replacing the liquid electrolyte with a gel or solid-type electrolyte, and better long-term stability is also expected. Further improving the environmental durability against heat and UV light is the next step toward practical applications.

\section{Acknowledgments}

The authors wish to thank RiTdisplay Corporation, Hsinchu Industrial Park, Taiwan, for providing the conductive ITO glass substrates. This research was 
supported by the National Research Council of the Republic of China under Grants NSC92-2214-E002-037 and NSC92-2622-E002-033-CC3. We also appreciate the support received from the ITRI and the NTU-ITRI Nano Center.

\section{References}

[1] C.G. Granqvist, A. Azens, A. Hjelm, L. Kullman, G.A. Niklasson, D. Rönnow, M.S. Mattsson, M. Veszelei, G. Vaivars, Sol. Energy Mater. Sol. Cells 63 (1998) 199-216.

[2] C.G. Granqvist, A. Azens, J. Isidorsson, M. Kharrazi, L. Kullman, T. Lindström, G.A. Niklasson, C.G. Ribbing, D. Rönnow, M.S. Mattsson, M. Veszelei, J. Non-Cryst. Solids 218 (1997) 273-279.

[3] C.G. Granqvist, E. Avendaño, A. Azens, Thin Solid Films 442 (2003) 201-211.

[4] M. Mastragostino, C. Arbizzani, A. Bongini, G. Barbarella, M. Zambianchi, Electrochim. Acta 38 (1993) 135-140.

[5] J. Roncali, Chem. Rev. 92 (1992) 711-738.

[6] M. Mastragostino, A.M. Marinangeli, A. Corradini, S. Giacobbe, Synth. Methods 28 (1989) C501-C506.

[7] F.J. Pern, A.J. Frank, J. Electrochem. Soc. 137 (1990) 2769-2777.

[8] A. Corradini, A.M. Marinangeli, M. Mastragostino, Electrochim. Acta 35 (1990) 1757-1760.

[9] C. Arbizzani, M. Mastragostino, S. Passerini, R. Pileggi, B. Scrosati, Electrochim. Acta 36 (1991) 837-840.

[10] C. Arbizzani, M. Mastragostino, L. Meneghello, M. Morselli, A. Zanelli, J. Appl. Electrochem. 46 (1996) 121-123.

[11] A.S. Ribeiro, D.A. Machado, P. Faria dos Santos Filho, M.A. De Paoli, J. Electroanal. Chem. 567 (2004) 243-248.

[12] F. Garnier, G. Tourillon, J. Electroanal. Chem. 148 (1983) 299-303.

[13] G. Shi, S. Jin, G. Xue, C. Li, Science 267 (1995) 994-996.

[14] S. Alkan, C.A. Culter, J.R. Reynolds, Adv. Funct. Mater. 13 (2003) 331-336.

[15] D. Zhang, J. Qin, G. Xue, Synth. Methods 106 (1999) 161-164.

[16] D. Zhang, J. Qin, G. Xue, Synth. Methods 100 (1999) 285-289.

[17] S.A. Sapp, G.A. Sotzing, J.R. Reynolds, Chem. Mater. 10 (1998) 2101-2108.

[18] A. Kros, S.W.F.M. Van Hovell, N.A.J.M. Sommerdijk, R.J.M. Nolte, Adv. Mater. 13 (2001) $1555-1557$.

[19] S. Fabiano, C. Tran-Minh, B. Piro, L.A. Dang, M.C. Pham, O. Vittori, Mater. Sci. Eng. C 21 (2002) 61-67.

[20] Y. Kudoh, K. Akami, Y. Matsuya, Synth. Methods 1-3 (1999) 973-974.

[21] Y. Saito, T. Kitamura, Y. Wada, S. Yanagida, Synth. Methods 131 (2002) 185-187.

[22] X. Chen, O. Inganäs, J. Phys. Chem. 100 (1996) 15202-15206.

[23] G.L. Gaupp, D.M. Welsh, R.D. Rauh, J.R. Reynolds, Chem. Mater. 14 (2002) 3964-3970. 